
The Eightfold Way

MSRI Publications

Volume 35, 1998

\section{From the History of a Simple Group}

\section{JEREMY GRAY}

The attractive pattern of 168 shaded and 168 unshaded triangles shown in Figure 1 has an interesting history. Since its discovery by Klein in 1878 (see

Figure 1

This article originally appeared in The Mathematical Intelligencer 4:2 (1982), 59-67. The editor is thankful to the author and to Springer-Verlag for permission to reprint. 
[Klein 1879]), it has often been reproduced; a close cousin (Figure 2) inspired the badge of the 1978 International Congress of Mathematicians in Helsinki. This article considers its origins, which lie in the fields of nineteenth century geometry and the theory of equations.

But first let us look closely at the figure itself. Each triangle, shaded or unshaded, has angles of $\pi / 2, \pi / 3$, and $\pi / 7$. Since

$$
\frac{\pi}{2}+\frac{\pi}{3}+\frac{\pi}{7}=\frac{41 \pi}{42}<\pi
$$

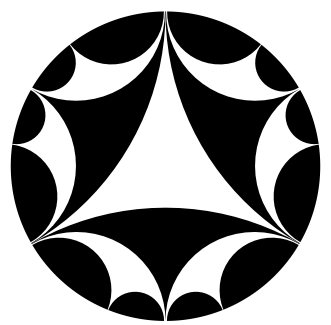

Figure 2

we immediately recognize that this is a non-Euclidean figure, but we shall see that Klein missed this conclusion altogether.

In each of the 14 slices emanating from the center there are 12 shaded and 12 unshaded triangles, so there are 168 of each kind. The sides of each triangle are arcs of circles orthogonal to the boundary circles, or are diameters. The figure can be continued in this fashion to reach indefinitely close to the boundary, and it provides in this way a tessellation of the non-Euclidean plane. The unshaded tessellation is preserved by non-Euclidean reflection in any side of any triangle (i.e., by inversion) and so has the group of all such reflections as its symmetry group. The group generated by all products of pairs of reflections is the symmetry group of the shaded figure.

Klein had been led to construct the figure because of its use in studying a certain polynomial equation (described at the end of this paper) for which the group permuting the roots is $\operatorname{PSL}(2 ; \mathbb{Z} / 7 \mathbb{Z})$, sometimes known as $G_{168}$ because of the number of its elements. Our first task, then, is to understand this group geometrically.

The $\operatorname{map} \mathbb{Z} \rightarrow \mathbb{Z} / 7 \mathbb{Z}$ which takes residues modulo 7 induces a homomorphism between two groups of $2 \times 2$ matrices:

$$
\mathrm{SL}(2 ; \mathbb{Z}) \rightarrow \mathrm{SL}(2 ; \mathbb{Z} / 7 \mathbb{Z})
$$

where $\mathrm{SL}(2, K)$ is the group of $2 \times 2$ matrices with entries in $K$ and of determinant 1. This is an onto map, and we shall denote its kernel by $\Gamma_{7}$. The group $\operatorname{SL}(2 ; \mathbb{Z})$ acts on the upper half-plane $H=\{z \in \mathbb{C}: \operatorname{Im}(z)>0\}$ : the element $\left(\begin{array}{ll}a & b \\ c & d\end{array}\right)$ in $\operatorname{SL}(2 ; \mathbb{Z})$ sends $z$ to $(a z+b) /(c z+d)$.

Since $\left(\begin{array}{ll}a & b \\ c & d\end{array}\right)$ and $\left(\begin{array}{ll}-a & -b \\ -c & -d\end{array}\right)$ have the same effect on all $z \in H$, it is sometimes convenient to factor out the centre, $\{ \pm 1\}$, of $\mathrm{SL}(2 ; \mathbb{Z})$, and obtain $\operatorname{PSL}(2 ; \mathbb{Z})=$ $\mathrm{SL}(2 ; \mathbb{Z}) /\{ \pm 1\}$, which acts faithfully on $H$. Dedekind was the first to describe this group geometrically, in a very important paper [1878]. He defined the region

$$
R=\left\{z \in H:|z| \geq 1,-\frac{1}{2} \leq \operatorname{Re}(z) \leq \frac{1}{2}\right\}
$$

(see Figure 3), and showed that the orbit

$$
O(z)=\left\{\frac{a z+b}{c z+d}:\left(\begin{array}{ll}
a & b \\
c & d
\end{array}\right) \in \mathrm{SL}(2 ; \mathbb{Z})\right\}
$$




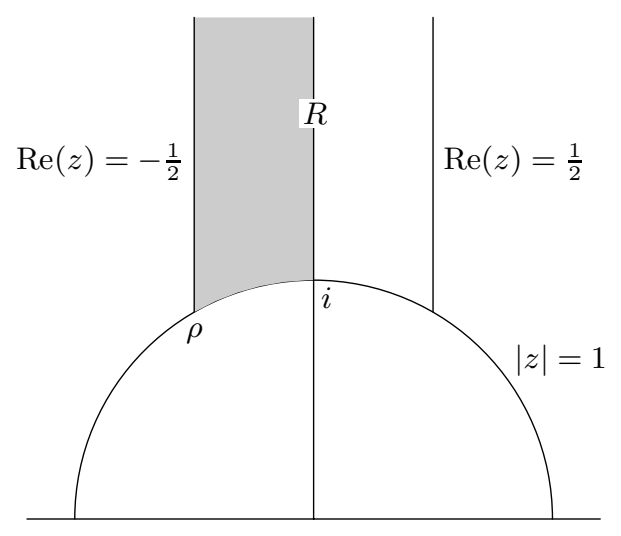

Figure 3

of each point $z$ of $H$ meets $R$ precisely once (in its interior) or twice (on its boundary). Consequently $\mathrm{SL}(2 ; \mathbb{Z})$ moves this region around en bloc, and covers $H$ like a tile, with overlaps occurring only on copies of the boundary of $R$. Moreover, as Dedekind said, the elements of $\operatorname{SL}(2 ; \mathbb{Z})$ are all products of these matrices:

$$
\begin{aligned}
& \left(\begin{array}{rr}
0 & -1 \\
1 & 0
\end{array}\right), \quad \text { which sends } z \text { to }-1 / z \text { and fixes } i \text {, and } \\
& \left(\begin{array}{rr}
0 & -1 \\
1 & 1
\end{array}\right), \quad \text { which sends } z \text { to }-1 /(z+1) \text { and fixes } \rho,
\end{aligned}
$$

a cube root of unity.

Klein took over Dedekind's simple geometric presentation, and refined it by making explicit that the set of all $2 \times 2$ matrices with integer entries and determinant 1 is a group, a fact Dedekind had not stressed although he would have been well aware of it, and by looking for particular subgroups of it. The one of most interest to him was $\Gamma_{7}$.

The index of $\Gamma_{7}$ in $\operatorname{SL}(2 ; \mathbb{Z})$ is, of course, the order of $\operatorname{SL}(2 ; \mathbb{Z} / 7 \mathbb{Z})$. Since Galois's work had been published (in 1846) it had been usual to consider the action of this group on the eight symbols $0,1, \ldots, 7, \infty$ by

$$
z \mapsto \frac{\alpha z+\beta}{\gamma z+\delta}, \quad\left(\begin{array}{ll}
\alpha & \beta \\
\gamma & \delta
\end{array}\right) \in \operatorname{SL}(2 ; \mathbb{Z} / 7 \mathbb{Z}) .
$$

These symbols can be regarded as the slopes of lines through the origin in the plane defined over the field of 7 elements (more precisely, as the points of the projective line over that field). So the group $\mathrm{SL}(2 ; \mathbb{Z} / 7 \mathbb{Z})$ has 336 elements, for there are 8 directions for the position of the first basis vector $\left(\begin{array}{l}1 \\ 0\end{array}\right)$ under $\left(\begin{array}{ll}\alpha & \beta \\ \gamma & \delta\end{array}\right)$, each with 6 possible positions for the image of $\left(\begin{array}{l}1 \\ 0\end{array}\right)$ itself, then there are 7 choices for the direction of the image of $\left(\begin{array}{l}1 \\ 0\end{array}\right)$, but no choice for its position once the direction is chosen, since $\alpha \delta-\beta \gamma=1: 8 \cdot 6 \cdot 7=336$. The group $\operatorname{PSL}(2 ; \mathbb{Z} / 7 \mathbb{Z})$ therefore has $\frac{1}{2} 336=168$ elements. So, looking at the faithful action, one finds 
that $\bar{\Gamma}_{7}=\Gamma_{7} /\{ \pm 1\}$ has index 168 in $\operatorname{PSL}(2 ; \mathbb{Z})$. So it must move 168 copies of $R$ around en bloc, and a suitable choice of which 168 copies can be made depending on the purposes at hand. One way is to observe that

$$
\left(\begin{array}{rr}
0 & -1 \\
1 & 0
\end{array}\right)\left(\begin{array}{rr}
0 & -1 \\
1 & 1
\end{array}\right)=\left(\begin{array}{rr}
-1 & -1 \\
0 & -1
\end{array}\right)
$$

which is equivalent to $\left(\begin{array}{ll}1 & 1 \\ 0 & 1\end{array}\right)$ in $\operatorname{PSL}(2 ; \mathbb{Z})$, an element which has the effect of shifting the triangle sideways by $z \mapsto z+1$. Since

$$
\left(\begin{array}{ll}
1 & 1 \\
0 & 1
\end{array}\right)^{n}=\left(\begin{array}{ll}
1 & n \\
0 & 1
\end{array}\right)
$$

the element

$$
\left(\begin{array}{ll}
1 & 1 \\
0 & 1
\end{array}\right)^{7}
$$

is in $\bar{\Gamma}_{7}$, so one picks 7 copies of $R$ in a horizontal strip. One can also pick other elements of $\bar{\Gamma}_{7}$ which yield other copies of $R$, until the 168 block is determined.

To study the quotient, $G_{168}$, one observes that any matrix representative of an element in it also moves the 168-member block around, but that action is only defined modulo $\bar{\Gamma}_{7}$, so $G_{168}$ really maps the 168 -member block to itself, once suitable identifications have been made. The case $G_{168}$ is rather unwieldy at first glance, so consider for a moment starting with residues modulo 2 :

$$
\bar{\Gamma}_{2} \rightarrow \operatorname{PSL}(2 ; \mathbb{Z}) \rightarrow \operatorname{PSL}(2 ; \mathbb{Z} / 2 \mathbb{Z}) .
$$

$\operatorname{PSL}(2 ; \mathbb{Z} / 2 \mathbb{Z})$ has only 6 elements:

$$
\left(\begin{array}{ll}
1 & 0 \\
0 & 1
\end{array}\right),\left(\begin{array}{ll}
1 & 1 \\
0 & 1
\end{array}\right),\left(\begin{array}{ll}
0 & 1 \\
1 & 0
\end{array}\right),\left(\begin{array}{ll}
0 & 1 \\
1 & 1
\end{array}\right),\left(\begin{array}{ll}
1 & 1 \\
1 & 0
\end{array}\right),\left(\begin{array}{ll}
1 & 0 \\
1 & 1
\end{array}\right)
$$

So $\bar{\Gamma}_{2}$ moves 6 copies of $R$ around en bloc, and $\operatorname{PSL}(2 ; \mathbb{Z} / 2 \mathbb{Z})$ can be regarded as a group of self-maps of that region. The most attractive picture of this is shown in Figure 4, and one notices that the action of $\bar{\Gamma}_{2}$, unlike that of $\operatorname{PSL}(2 ; \mathbb{Z})$, is fixed-point free. It is this figure that inspired the ICM badge.

The action of $\bar{\Gamma}_{2}$ identifies the edges of the larger region in pairs, and so one can ask what the region is topologically. In this case it is clear from the identifications that the region is a sphere.

There is one problem with these pictures: the vertex at $\infty$ of the region $R$. Klein simply switched to a region $\tilde{R}$ where this angle was $2 \pi / 7$, since $\bar{\Gamma}_{7}$ cycles 7 copies of $R$ around the vertex. This can be done by standard moves in the theory of complex functions: either appeal to the Riemann mapping theorem, or find an explicit map of $R$ holomorphic everywhere except at copies of $\infty$, where it has a suitable branch point. Klein presumably did the first; subsequently a student of his, the American mathematician M. W. Haskell, did the second using a quotient of two solutions to a hypergeometric equation [Haskell 1891]. Finally we have Figure 1 before us, together with a description of $G_{168}$ as the self-maps 


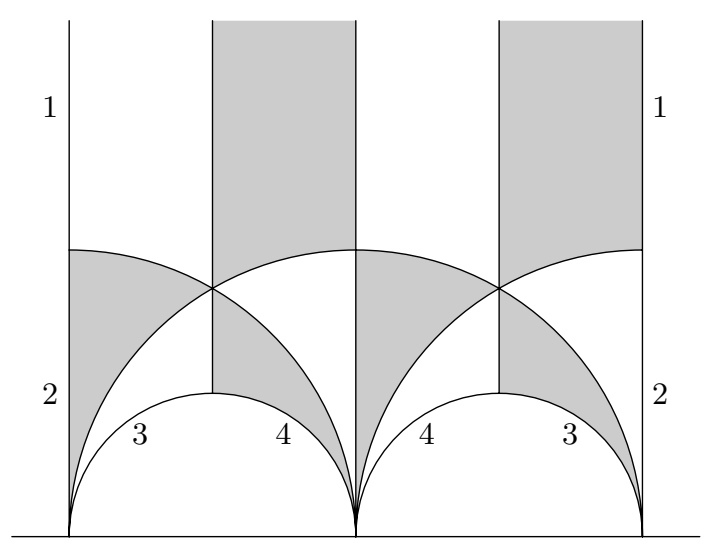

Figure 4

of this region, thought of as the quotient space $\bar{\Gamma}_{7} \backslash H$, which also preserve the shading. It turns out that the edge identifications are 1 with 6,3 with 8 and so on, and the even- and odd-numbered edges are directed oppositely so that the triangles match up.

Klein was interested in the figure as an algebraic curve and as a Riemann surface so he wanted to know its genus. This can be found using Euler's formula

$$
V-E+F=2-2 g
$$

where $V, E$, and $F$ denote the number of vertices, edges and faces in a triangulation of the surface, and $g$ is the genus.

Happily, we have a triangulation: it has 336 triangles (since $\bar{R}$ is made up of 2 triangles), so $F=336$; and $E=336 \cdot 3 / 2$, since each edge is counted twice. As for the vertices, 336 have angles of $\pi / 7$, so 14 cluster together at each one; 336 have angles of $\pi / 3$ and are identified in 6's; and 336 have angles of $\pi / 2$ and are identified in 4's: a total of

$$
336\left(\frac{1}{14}+\frac{1}{6}+\frac{1}{4}\right)=164
$$

so $V-E+F=4$, and $g=3$ : the Riemann surface has genus 3 . Klein followed Riemann's approach of looking at the order of the branch points in order to calculate the genus.

It then follows from Riemann's work on algebraic curves $[1857, \S 13]$ that the surface must be describable as a quartic, that is, using homogeneous coordinates $[x, y, z]$, by a homogeneous polynomial of degree 4 . Klein then turned to the projective theory of higher plane curves that had been developed in the preceding generation, and showed how it could be illuminated by his new methods of Riemann surface theory. This is a path well worth following. 


\section{Plane Algebraic Curves}

Although Newton had provided a very thorough analysis of cubics in the 1670's, mathematicians rather neglected the systematic study of algebraic curves other than conics until the start of the nineteenth century. When finally they began, they confronted the question of deciding what could interestingly be said about the profusion of new cubics, quartics, quintics, and so forth with which they were confronted. The properties that most attracted them were projective in nature, and were not shared by conics, notably: points of inflection, bitangents (lines tangent at two places to a curve), double points, and cusps. The pioneer in this study was Plücker, who was Klein's first mathematical teacher. Plücker [1835] showed that a non-singular curve $F(x, y, z)=0$ of degree $n$ has $3 n(n-2)$ inflection points. Hesse's proof [1844] is simpler, being couched in homogeneous coordinates, but it essentially followed Plücker's argument. Hesse observed that at an inflection point adjacent normals are parallel, and so the mean curvature vanishes there. But the formula for the mean curvature is

$$
\left|\frac{\partial^{2} F}{\partial x_{i} \partial x_{j}}\right|
$$

which equated to zero is a curve of degree $3(n-2)$. So, by Bezout's principle, it meets $F=0$ in $3(n-2) n$ points, which are the points of inflection.

This result led Plücker to make an intriguing observation in his next book [1839]. The tangent to $F=0$ at $p$ has equation

$$
x_{1} \frac{\partial F}{\partial x_{1}}(p)+x_{2} \frac{\partial F}{\partial x_{2}}(p)+x_{3} \frac{\partial F}{\partial x_{3}}(p)=0
$$

and the triple

$$
\left(\frac{\partial F}{\partial x_{1}}(p), \frac{\partial F}{\partial x_{2}}(p), \frac{\partial F}{\partial x_{3}}(p)\right)
$$

can be thought of as defining the line coordinates of the tangent. This triple can be thought of as a point in the dual space to the original projective plane, and thus as defining a new plane curve called the dual of the original curve (see Figure 5). Geometrically this can be done by picking a circle and then replacing each point to the original curve by its polar with respect to the circle, and looking at the envelope of the polars. Both methods were used. What is the degree of the dual curve? Poncelet [1832] had shown that the tangents through $\left(\xi_{1}, \xi_{2}, \xi_{3}\right)$ to $F=0$ had equations

$$
\xi_{1} \frac{\partial F}{\partial x_{1}}(p)+\xi_{2} \frac{\partial F}{\partial x_{2}}(p)+\xi_{3} \frac{\partial F}{\partial x_{3}}(p)=0
$$

for suitable $P$ on $F=0$. The locus of all points in the plane for which this equation is true (for a given $\left[\xi_{1}, \xi_{2}, \xi_{3}\right]$ and $F$ ) is a curve of degree $n-1$ called a first polar of $F$. It meets $F=0$ in $n(n-1)$ points, so in general there are $n(n-1)$ tangents to a given curve of degree $n$ from a given point. Consequently 


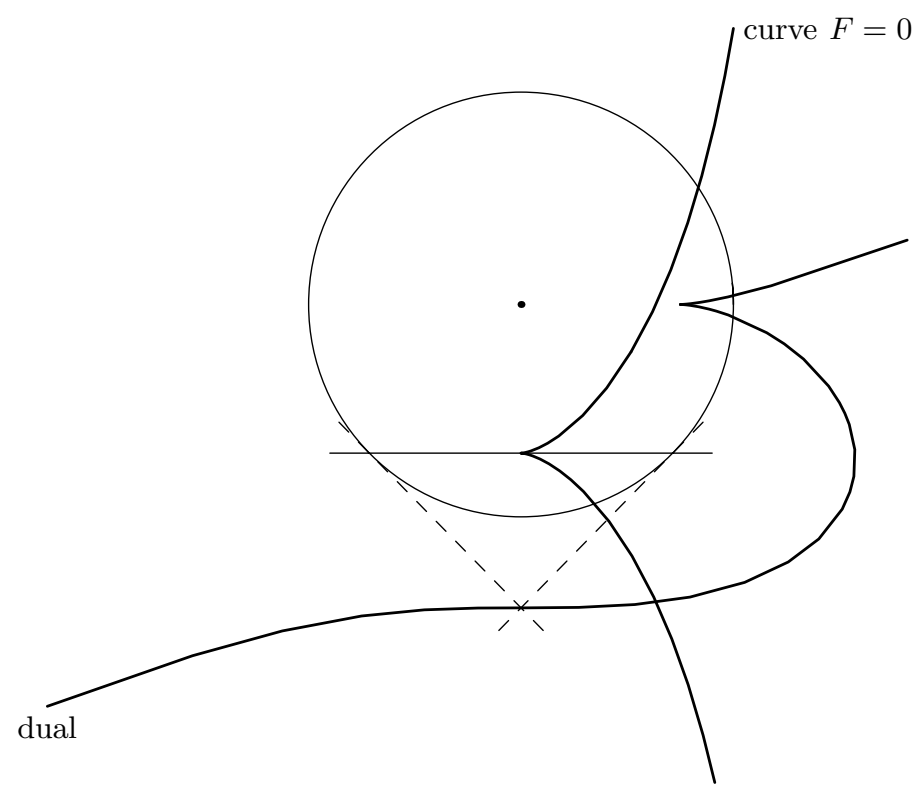

Figure 5

the dual curve to a curve of degree $n$ is of degree $n(n-1)$, for the dual of $n(n-1)$ lines through a point is $n(n-1)$ points on a line.

Plücker's intriguing paradox is this: plainly the dual curve of a dual curve is the original curve, but the degree formula shows that the double dual has degree $n(n-1)(n(n-1)-1)$, which is greater than $n$ as soon as $n>2$. Plücker had a solution: any line through a double point is a tangent, since it meets the curve in two coincident points, so the first polar passes through that point. But this is not what tangency is really about, and if those intersections are ignored by pulling the double point apart, this means two intersections of a curve and its first polar must be discussed. So each double point on the original curve lowers the degree of its dual by 2. Moreover, if the curve has a cusp the first polar is a tangent there, so each cusp lowers the degree by 3 . For example, the curve $x_{1}^{2} x_{3}-x_{2}^{3}=0$ has a cusp at $[0,0,1]$. Its first polar with respect to $[0,0,1]$ is $x_{1}^{2}=0$, which in fact is the equation of the tangent in this example.

So, if the original curve has $\alpha$ bitangents and $\beta$ inflection points, the dual will have $\alpha$ double points and $\beta$ cusps, since bitangents dualize to double points and inflection points to cusps. So if

$$
2 \alpha+3 \beta=n(n-1)(n(n-1)-1)-n=n^{3}(n-2),
$$

the paradox is explained. Moreover, Plücker had already shown that $\beta=3 n \times$ $(n-2)$, from which he deduced that

$$
\alpha=\frac{1}{2} n(n-2)\left(n^{2}-9\right),
$$


and he proclaimed that a nonsingular curve of degree $n$ has a dual with $\alpha$ double points, $\beta$ cusps, and degree $n(n-1)$, where

$$
2 \alpha+3 \beta=n^{3}(n-2)
$$

This formula is now called Plücker's formula. The value of $\alpha$ was first calculated directly by Jacobi [1850]. The particular case when $n=4$ is of most interest to us: a non-singular quartic should have 24 inflection points and 28 bitangents. The inflection points cannot all be real, but the bitangents can be, and Plücker even gave an example [1839]. He took two degenerate quartics: the four straight lines in Figure 6, and the circle counted twice. A linear combination of the equations of these two curves defines a quartic with double points at $(0,0),(1,1),(1,-1)$, and a vertical tangent at the points $a, a^{\prime}$. The figure shows in thin lines the particular combination

$$
\Omega_{4}=(y+x y)(y-x)(x-1)(x-1.85)-5\left(y^{2}+x(x-2)\right)^{2}=0 .
$$

When deformed into $\Omega_{4}-k=0$, for $k>0$ small, the curve splits apart into four bean-shaped pieces - the thick curves of the figure. Each has one bitangent of its own and each pair has 4 , a total of $4+6 \cdot 4=28$ in all. (We have varied

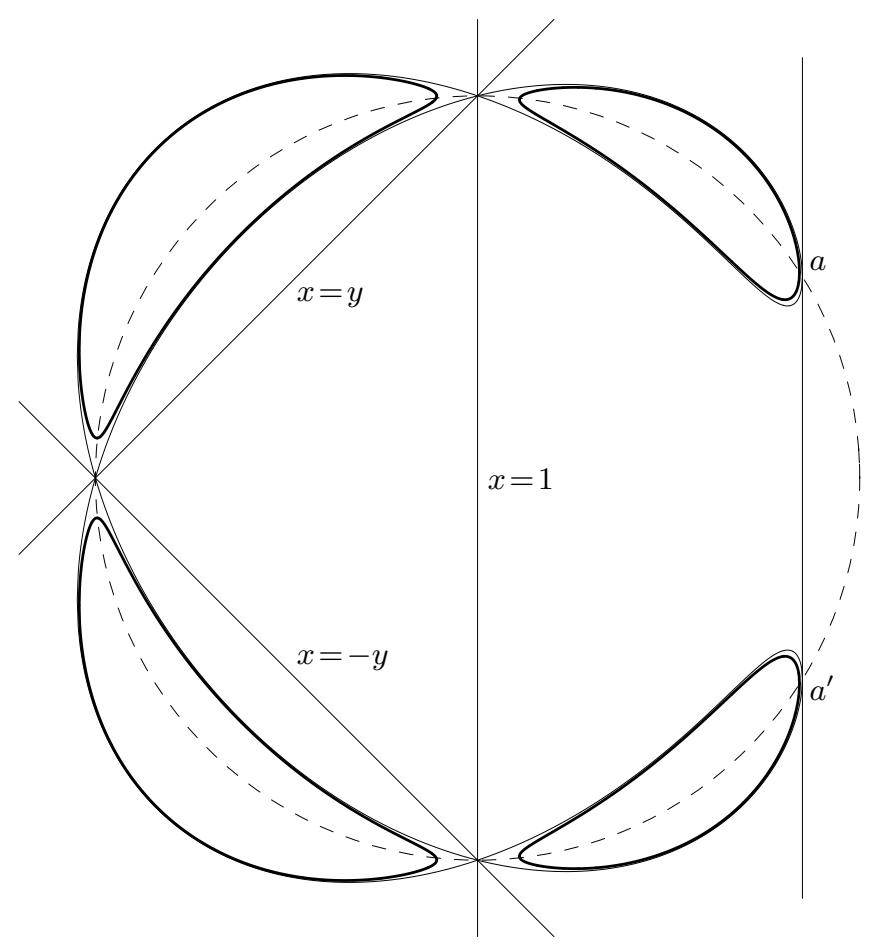

Figure 6 
Plücker's coefficients, which were $\frac{3}{2}$ and 2 instead of 1.85 and 5 , in order to make the concavity more apparent.)

The 28 bitangents became, and remain, a topic of delight. They are, for instance, intimately connected to the 27 lines on a cubic surface, a fact first noticed by Geiser [1869], and their automorphism group is isomorphic to the Weyl group of the exceptional Lie algebra $E_{7}$. Their history is far too lengthy to describe here, even in the period before Klein, but mention should be made of Hesse's paper [1855], in which he studied them via the 28 lines through 8 points in space, and in particular, to work of Riemann.

Riemann's "Theorie der Abelschen Functionen" introduced an (infinitely many valued) "function" $\theta$ of $p$ variables on a Riemann surface of genus $p$ which was crucial to his solution to the Jacobi inversion problem for integrals. He associated what he called a characteristic to $\theta$, an expression

$$
\left(\begin{array}{c}
e_{1}, e_{2}, \ldots, e_{p} \\
e_{1}^{\prime}, e_{2}^{\prime}, \ldots, e_{p}^{\prime}
\end{array}\right)
$$

where each entry is 0 or 1 , and he said the characteristic was odd if

$$
e_{1} e_{1}^{\prime}+e_{2} e_{2}^{\prime}+\cdots+e_{p} e_{p}^{\prime}
$$

was odd, and even otherwise. Induction on $p$ shows that $\left(2^{p-1}\right)\left(2^{p}-1\right)$ characteristics are odd.

When the characteristic is odd $\theta$ has two repeated zeros and 2 repeated poles on the surface, so it could be made to yield a bitangent curve to the surface, and when $p=3$ indeed to yield a bitangent. All this material, although partly published by Riemann, his student Roch, and by Clebsch, was very obscure to Riemann's contemporaries. Clebsch himself thought this was due to the elusive nature of the $\theta$-function, which was defined transcendentally and only after a long series of boldly innovative remarks. Riemann's paper defines Riemann surfaces and studies them topologically, uses the contentious Dirichlet principle to prove an index theorem for the genus, considers what functions can exist on a Riemann surface and proves the Riemann inequality for the dimension of the space of meromorphic functions with prescribed poles, discusses coordinate transformations and birational transformations of a given curve and the dimension of the corresponding moduli space of inequivalent curves of a given genus, and proves half of Abel's theorem before getting round to Jacobi inversion. Little wonder people found it difficult! But the notation of the characteristics was convenient, and in 1874 Weber used it to describe how the 28 bitangents are related (see [Weber 1876]).

Briefly, Steiner had shown in [1848] that the bitangents fell into 63 groupings of 6 pairs, with the property that the contact points of each pair with the quartic gave a set of 8 points lying on a conic. Weber showed that the 63 families could be indexed by the 63 characteristics other than $\left(\begin{array}{lll}0 & 0 & 0 \\ 0 & 0 & 0\end{array}\right)$ and that the sum of each 
pair of characteristics in a grouping was the indexing characteristic. Thus $\left(\begin{array}{lll}0 & 0 & 1 \\ 0 & 0 & 0\end{array}\right)$ indexes the pairs

$$
\begin{aligned}
& \left(\begin{array}{lll}
1 & 0 & 1 \\
1 & 0 & 0
\end{array}\right),\left(\begin{array}{lll}
1 & 0 & 0 \\
1 & 0 & 0
\end{array}\right) ; \quad\left(\begin{array}{lll}
0 & 1 & 1 \\
0 & 1 & 0
\end{array}\right),\left(\begin{array}{lll}
0 & 1 & 0 \\
0 & 1 & 0
\end{array}\right) ; \\
& \left(\begin{array}{lll}
1 & 1 & 1 \\
1 & 0 & 0
\end{array}\right),\left(\begin{array}{lll}
1 & 1 & 0 \\
1 & 0 & 0
\end{array}\right) ;\left(\begin{array}{lll}
1 & 1 & 1 \\
0 & 1 & 0
\end{array}\right),\left(\begin{array}{lll}
1 & 1 & 0 \\
0 & 1 & 0
\end{array}\right) ; \\
& \left(\begin{array}{lll}
0 & 1 & 1 \\
1 & 1 & 0
\end{array}\right),\left(\begin{array}{lll}
0 & 1 & 0 \\
1 & 1 & 0
\end{array}\right) ;\left(\begin{array}{lll}
1 & 0 & 1 \\
1 & 1 & 0
\end{array}\right),\left(\begin{array}{lll}
1 & 0 & 0 \\
1 & 1 & 0
\end{array}\right)
\end{aligned}
$$

and

$$
\left(\begin{array}{lll}
1 & 0 & 1 \\
1 & 0 & 0
\end{array}\right)+\left(\begin{array}{lll}
1 & 0 & 0 \\
1 & 0 & 0
\end{array}\right)=\left(\begin{array}{lll}
0 & 0 & 1 \\
0 & 0 & 0
\end{array}\right),
$$

etc. (adding coordinatewise, mod 2). This approach was in fact that of Riemann [1862], as Weber found out on becoming joint editor of Riemann's Werke with Dedekind later in 1874, and had earlier been taken by Clebsch [1864], which Weber seems not to have known. The geometric situation is that two conics, each touching a quartic in 4 points, lie in the same system if their 8 points lie in a conic. There are 63 systems (a result of Hesse's) and each system contains 6 line pairs, the pairs of bitangents.

Jordan, basing himself on Clebsch's work - which was published, rather than on Riemann's, which was not - gave an analysis of the 28 bitangents in [Jordan 1870]. He showed ( $\S 332$ ) that the group of symmetries of the bitangents is isomorphic to the symplectic group $\operatorname{Sp}(6 ; \mathbb{Z} / 2 \mathbb{Z})$, that is, to the group of $6 \times 6$ matrices over the field of 2 elements which preserves the inner product represented by the matrix

$$
A=\left(\begin{array}{rr}
0 & I \\
-I & 0
\end{array}\right) \equiv\left(\begin{array}{ll}
0 & I \\
I & 0
\end{array}\right)(\bmod 2),
$$

where $I$ is the $3 \times 3$ identity matrix. He also showed ( $\S 455)$ that the subgroup of the group of symmetries which fixes a bitangent is isomorphic to the group of symmetries of the 27 lines in a cubic surface, thereby connecting his work to Geiser's.

It is clear to us that each characteristic is a vector in the six-dimensional vector space over $\mathbb{Z} / 2 \mathbb{Z}$ and that the bitangents corresponding to those vectors $\boldsymbol{v}$ for which $\boldsymbol{v}^{T} A \boldsymbol{v} \equiv 1(\bmod 2)$; and, moreover, the action of $G_{168}$ in its alternative guise as $\mathrm{SL}(3 ; \mathbb{Z} / 2 \mathbb{Z})$ is also now apparent. This version of $G_{168}$ was presented by Weber in [1896, p. 539], where he attributed it to Kronecker. However, Jordan did not use this geometric approach, nor did Dickson in his discussion [1900]. It would be interesting to know who first interpreted the characteristics in terms of finite geometries, thereby making explicit what was implicit, but not geometric, in [Jordan 1870]. The American mathematician A. B. Coble [1908; 1913] seems to have been the first to illuminate the 27 lines and 28 bitangents with the elementary theory of geometries over finite fields.

The combinatorial aspects of all this are pleasant, but the mathematics is certainly not easy. All the more attractive then for Klein when he saw how to make some of these configurations visible in this picture of his Riemann surface. 


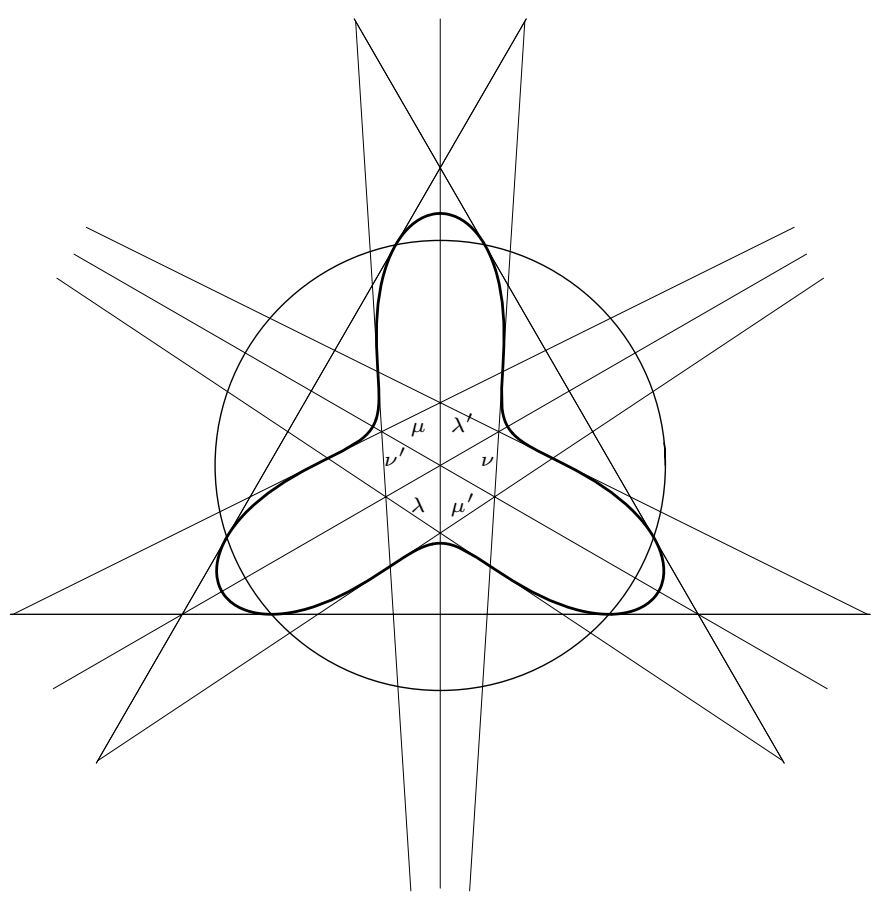

Figure 7. From [Klein 1879].

Let us return to the description of the surface as an algebraic curve. It is a quartic, and Klein showed quickly that if three suitable inflection tangents are taken as triangle of reference the equation can be written as

$$
F\left(x_{1}, x_{2}, x_{3}\right)=x_{1}^{3} x_{2}+x_{2}^{3} x_{3}+x_{3}^{3} x_{1}=0 .
$$

As a real locus it looks like the curve in Figure 7 . Under the action of $G_{168}$ a typical point has an orbit consisting of 168 points. But some have smaller orbits: the vertices have orbits consisting of 24,56 , and 84 points. These must, by simple considerations of invariant theory, said Klein, correspond to the 24 inflection points, the 56 bitangents points, and 84 sextatic points (where a conic has sixfold contact with the curve). So there are all these points, hitherto hard to visualize, all laid out in one figure. Klein called them $\boldsymbol{a}, \boldsymbol{b}$ and $\boldsymbol{c}$ points respectively.

To get at the inter-relations of these points, Klein used elementary matrix algebra and group theory to give an exhaustive analysis of the subgroups of $G_{168}$. He found, amongst other subgroups, 14 of order 4 (now called Klein's group), 14 of order 24, which come in two families of 7 conjugates, and 28 nonabelian groups of order 6 . The analysis showed that $G_{168}$ has no non-trivial normal subgroups, but Klein did not remark explicitly on its simplicity. Indeed, he was more interested in the existence of 8 conjugate subgroups of order 21 , 
and of two families of 7 conjugate subgroups of order 24 which are isomorphic to the group of proper motions of an octahedron.

It is an easy matter to carry out the matrix algebra to find all the elements of a given order. For example, elements of order 2 must have vanishing trace. One can then arrange them in conjugacy classes, and thence find all the subgroups of a given order. But it is not so easy to see them in the figure, because the action of the group is not so clear and it is difficult to work with the identifications. Klein argued as follows. The $\boldsymbol{b}$-points, for example, are each fixed by a rotation of order 3 . There are 28 groups of order 3 in $G_{168}$, so each such group fixes a pair of $\boldsymbol{b}$-points and these are the points of contact of a bitangent. The $\boldsymbol{c}$-points are fixed by rotations of order 2 , of which there are 21 , so each such rotation fixes 4 of them.

To display them in the figure, Klein considered the 28 "symmetry lines" which run in a cycle through six $\boldsymbol{a}, \boldsymbol{b}$, and $\boldsymbol{c}$-points, as, for example, do the lines, which run straight from the central point. They must be pursued with care across the identifications, especially at the two kinds of vertex. There are 2 symmetry lines through each $\boldsymbol{c}$-point. There is then a unique pair of such lines which does not meet the first pair, and the $4 \boldsymbol{c}$-points so picked out are a typical set of $4 \boldsymbol{c}$-points fixed by a rotation. Similarly the 3 symmetry lines through a $\boldsymbol{b}$-point meet again in the corresponding $\boldsymbol{b}$-point. Klein denoted 4 such pairs in the figure by $A, A^{\prime}$; $B, B^{\prime} ; C, C^{\prime} ; D, D^{\prime}$. However, he was not able to go further with this analysis and exhibit the 63 systems of 6 pairs of bitangents. The Riemannian theory of theta-characteristics is not taken up in this paper, nor in the famous study [Klein 1882], and one rather supposes that Klein, like his contemporaries, did not really understand it.

But he did have other, new, things to say. He showed that $G_{168}$ could be written down as a group acting on complex projective two-space, since it was the automorphism group of a plane projective curve, and he exhibited its generators explicitly. This gave him a finite subgroup of $\mathrm{SL}(3 ; \mathbb{C})$, and he observed that it was missing from Jordan's list of such groups [Jordan 1878], which he had published earlier while studying differential equations all of whose solutions are algebraic. Jordan, a friend of Klein's since the latter's visit to Paris in 1870, accepted the correction in a letter and in a subsequent revision of his paper, but both men missed a presentation of $A_{6}$ as a subgroup of $\operatorname{SL}(3 ; \mathbb{C})$ subsequently found by Valentiner [1889] and named after him.

Finally, Klein gave a startling visual description of the Riemann surface. $G_{168}$ cannot be the symmetry group of a surface in space, but the subgroups of order 24 mentioned above can be, for each is the symmetry group of an octahedron. The octahedral group permutes the four pairs of diametrically opposite points in the middle of the faces of the octahedron, and Klein showed that the octahedral subgroups in $G_{168}$ permute 4 pairs such as $A, A^{\prime} ; B, B^{\prime} ; C, C^{\prime}$; and $D, D^{\prime}$. The $24 \boldsymbol{a}$-points may be taken as the centers of 24 heptagons, and when the figure is cut up along the six heavy zig-zag lines the heptagons may be taken in 3 's so 


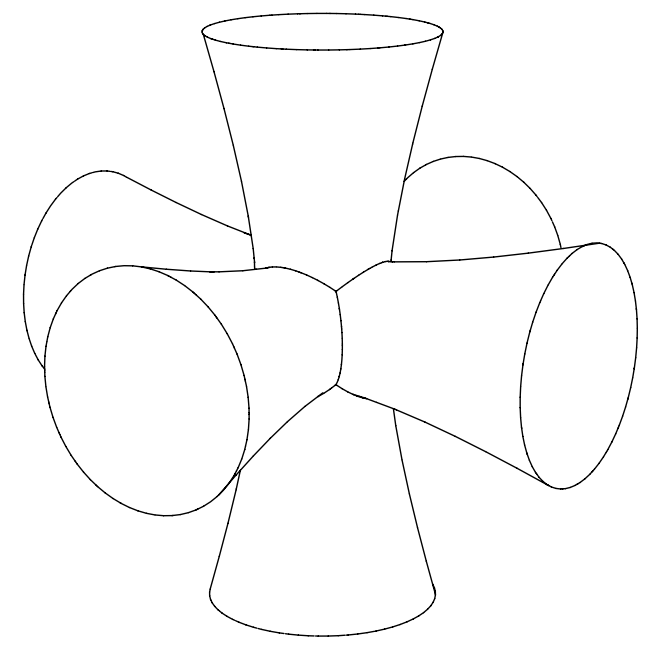

Figure 8

that each face of the octahedron is covered, more or less, with 21 shaded and 21 unshaded triangles. What is missing from this quilt is the six vertices of the octahedron. He regarded $G_{168}$ as acting partly by rotating the octahedron, and partly by sliding the quilt over the octahedron, using identifications across the edges surrounding the vertices, which come from this dissection. He showed that these identifications were of diametrically opposite points, and were best performed by supposing the edges drawn out to infinity. He invoked the analogy with the hyperboloid of one sheet, which, projectively, is a torus. The dotted curve in Figure 1 represents the intersections of the curve with the plane at infinity.

So he described the surface as three hyperboloids whose axes meet at right angles, which is certainly appealing (see Figure 8).

A few remarks should be made about why Klein studied this problem in the first place. An old problem in the theory of elliptic functions asks for a relation between the moduli of elliptic integrals if the corresponding ratios of the periods is increased by a prime $p$. Jacobi and Abel had shown that the moduli were then related by a polynomial equation of degree $p+1$. Galois know that the polynomial equation could be reduced in degree to an equation of degree $p$ when $p$ was 5,7 , and 11, but for no higher prime, and he know that for these equations the corresponding group permuting the roots (its "Galois" group, as we say, for this reason) was $\operatorname{PSL}(2, \mathbb{Z} / p \mathbb{Z})$. Various mathematicians, notably Betti [1853] and Jordan [1868], attributed this reduction to the existence of large subgroups (of index $p$ ) in $\operatorname{PSL}(2, \mathbb{Z} / p \mathbb{Z}$ ) when $p=5,7,11$, and showed that such subgroups did not exist for higher $p$. For example, when $p=7$, the group permutes its 7 conjugate octahedral subgroups, so it has a permutation representation of degree 7 . 
Dedekind's paper [1878] was devoted to establishing a theory of modular functions without recourse to the existing theory of elliptic functions, and central to it was a function val $: H \rightarrow \mathbb{C}$ (from the German Valenz) which takes each value once and only once on the interior of $R$ (and "half" its boundary) and for which

$$
\operatorname{val}(z)=\operatorname{val}\left(\frac{a z+b}{c z+d}\right) .
$$

Dedekind obtained it by taking the hypergeometric differential equation

$$
x(1-x) \frac{d^{2} y}{d x^{2}}+(\gamma-(\alpha+\beta+1) x) \frac{d y}{d x}-\alpha \beta y=0,
$$

where $x$ and $y$ are complex, and so choosing $\alpha, \beta$, and $\gamma$ that a quotient of the two solutions maps $\mathbb{C}$ on to $R$ or a copy of $R$ under the action of $\operatorname{SL}(2 ; \mathbb{Z})$. Then this function val is the inverse of this quotient.

Klein took over Dedekind's val function and renamed it $J$. He also took over (with due acknowledgement) Dedekind's theory of modular transformations, in which $J(z)$ and

$$
\tilde{J}(z)=J\left(\frac{A z+B}{C z+D}\right)
$$

are related, where $\left(\begin{array}{ll}A & B \\ C & D\end{array}\right)$ is a $2 \times 2$ matrix with integer entries and determinant $p$ (or, more generally, any natural number). It is not hard to see that if we regard as equivalent the elements

$$
\left(\begin{array}{ll}
A & B \\
C & D
\end{array}\right) \text { and }\left(\begin{array}{ll}
A & B \\
C & D
\end{array}\right)\left(\begin{array}{ll}
a & b \\
c & d
\end{array}\right),
$$

where $\left(\begin{array}{ll}a & b \\ c & d\end{array}\right) \in \mathrm{SL}(2 ; \mathbb{Z})$, then there are $p+1$ inequivalent $\left(\begin{array}{ll}A & B \\ C & D\end{array}\right)$ of determinant $p$. So the equation relating $J$ and $\tilde{J}$ is a polynomial equation of degree $p+1$ : the modular equation. Klein's contribution lay in making the groups explicit, and, more importantly, in introducing the Riemann surface of $\tilde{J}$ spread out over the complex $J$-sphere. Thus when $p=5$ a sphere is obtained, corresponding to the role of $\operatorname{PSL}(2 ; \mathbb{Z} / 5 \mathbb{Z})$ as the icosahedral group, and we have seen detail how Klein treated the next case, $p=7$. Indeed his famous book on the icosahedron [Klein 1884] is a showcase of his ideas on mathematics at the time, and his treatment of the quartic curve marks a high point in his style.

Rational functions in $J$ are quotients of polynomials in $J$, and form a field $\mathbb{C}(J)$. Rational functions in $\tilde{J}$ live on the Riemann surface for $\tilde{J}$, and form a field extension of $C(J)$ whose Galois group is the group of the modular equation. This work of Klein's is thus at the origin of the Galois theory of function fields. Gordan's jocular name for this kind of mathematics was, Klein tells us, hyperGalois theory [Klein 1922, p. 261]. The Galois-theoretic point of view is slightly further developed in Klein and Fricke's two-volume work on modular functions [Klein and Fricke 1890-92]. 
Dedekind had been the first in Germany to lecture on Galois theory, and the first to stress the importance of the concept of an abstract group (in lectures around 1858; see [Purkert 1976]), but he chose not to stress these ideas here, and it was left to Klein and his students to develop them.

Finally, why did Klein not notice the connection with non-Euclidean geometry? There can never be a simple answer to this question, but Klein's preferences were for projective geometry, and for using group theory to get at the invariant configurations (inflection points, bitangent points, and so on). In the paper analysed here, and in his work on the icosahedron, he succeeded brilliantly in his chosen task. The differential-geometric approach to non-Euclidean geometry advanced by Beltrami was less congenial to him, less central to his view of mathematics. So he did not look for such aspects of his problem, and the simple realization was left to Poincaré - to dramatic effect. Nonetheless, as Klein tells us [1923, p. 584], it was during a sleepless night, March 22-23, 1882, that Klein, in contemplating Figure 1, was able to grasp the full generality of Poincaré's ideas and so to formulate his own approach to automorphic functions and the uniformization of Riemann surfaces.

\section{References}

[Betti 1853] E. Betti, "Sopra l'abbassamento delle equazioni modulari delle funzioni ellittiche", Annali di scienze mat. e fis. (comp. da Barnaba Tortolini) 4 (1853), 81-100.

[Clebsch 1864] A. Clebsch, "Ueber die Anwendung der Abelschen Functionen in der Geometrie", J. für reine angew. Math. 63 (1864), 189-243.

[Coble 1908] A. Coble, "A configuration in finite geometry isomorphic with that of the 27 lines on a cubic surface", Johns Hopkins University Circular 7:80-88 (1908), 736-744.

[Coble 1913] A. Coble, "An application of finite geometry to the characteristic theory of the odd and even theta functions", Trans. Amer. Math. Soc. 14 (1913), 241-276.

[Dedekind 1878] R. Dedekind, "Schreiben an Herrn Borchardt über die Theorie der elliptischen Modulfunctionen", J. für reine angew. Math. 83 (1878), 265-292. Reprinted as pp. 174-201 in his Gesammelte mathematische Werke, vol. 1, Vieweg, Braunschweig, 1930; reprinted by Chelsea, 1969.

[Dickson 1900] L. E. Dickson, Linear Groups, with an exposition of the Galois Field Theory, Chicago, Leipzig, 1900. Reprinted by Dover, New York, 1958.

[Geiser 1869] C. F. Geiser, "Ueber die Doppeltangenten einer ebenen Curve vierten Grades", Math. Annalen 1 (1869), 129-138.

[Haskell 1891] M. W. Haskell, "Ueber die zu der Curve $\lambda^{3} \mu+\mu^{3} \nu+\nu^{3} \lambda=0$ im projectiven Sinne gehörende mehrfache Ueberdeckung der Ebene", Amer. J. Math. 13 (1891), 1-52.

[Hesse 1844] L. O. Hesse, "Ueber die Wendepunkte der Curven dritter Ordnung", J. für reine angew. Math. 28 (1844), 97-106. Reprinted as pp. 123-136 of his Gesammelte Werke, Chelsea, New York, 1972. 
[Hesse 1855] L. O. Hesse, "Ueber die Doppeltangenten der Curven vierter Ordnung", J. für reine angew. Math. 49 (1855), 279-332. Reprinted as pp. 345-404 of his Gesammelte Werke, Chelsea, New York, 1972.

[Jacobi 1850] C. G. J. Jacobi, "Beweis des Satzes, daß eine Curve $n^{\text {ten }}$ Grades in Allgemeinen $\frac{1}{2} n(n-2)\left(n^{2}-9\right)$ Doppeltangenten hat", J. für reine angew. Math. 40 (1850), 237-260. Reprinted as pp. 517-542 of his Gesammelte Werke, vol. 3, Gauthier-Villars, Paris, 1884.

[Jordan 1868] M. C. Jordan, "Note sur les équations modulaires", C. Rendus Acad. Sci. Paris 66 (1868), 308-312. Reprinted as pp. 159-163 of his Euvres, vol. 1, GauthierVillars, Paris, 1961.

[Jordan 1870] M. C. Jordan, Traité des substitutions et des équations algébriques, Gauthier-Villars, Paris, 1870.

[Jordan 1878] M. C. Jordan, "Mémoire sur les équations différentielles linéaires à intégrale algébrique", J. für reine angew. Math. 84 (1878), 89-215. Reprinted as pp. 13-140 of his Euvres, vol. 2, Gauthier-Villars, Paris, 1962.

[Klein 1879] F. Klein, "Ueber die Transformationen siebenter Ordnung der elliptischen Funktionen", Math. Annalen 14 (1879), 428-471. Reprinted as [Klein 1923, LXXXIV, pp. 90-136]. Translated in this collection.

[Klein 1882] F. Klein, Ueber Riemanns Theorie der algebraischen Funktionen und ihrer Integrale, Teubner, Leipzig, 1882. Reprinted as Reprinted as [Klein 1922, XCIX, pp. 499-573] Translated as On Riemann's Theory of Algebraic Functions and their Integrals, Macmillan and Bowes, Cambridge, 1893; reprinted by Dover, New York, 1963.

[Klein 1884] F. Klein, Vorlesungen über das Ikosaeder und die Auflösung der Gleichungen vom fünften Grade, Teubner, Leipzig, 1884. Translated as Lectures on the icosahedron and the solution of equations of the fifth degree, Kegan Paul, London, 1913 (2nd edition); reprinted by Dover, 1953.

[Klein 1922] F. Klein, Gesammelte Mathematische Abhandlungen, 2: Anschauliche Geometrie; Substitutionsgruppen und Gleichungstheorie; zur mathematischen Physik, edited by R. Fricke and H. Vermeil, Springer, Berlin, 1922. Reprinted by Springer, 1973.

[Klein 1923] F. Klein, Gesammelte Mathematische Abhandlungen, 3: Elliptische Funktionen etc., edited by R. Fricke et al., Springer, Berlin, 1923. Reprinted by Springer, 1973.

[Klein and Fricke 1890-92] F. Klein, Vorlesungen über die Theorie der elliptischen Modulfunctionen, ausgearbeitet und vervollständigt von Robert Fricke (2 vol.), Teubner, Leipzig, 1890-92. Reprinted by Johnson Reprint, New York, 1966.

[Plücker 1835] J. Plücker, System der analytischen Geometrie, auf neue betrachtungsweisen gegrundet. .., Duncker und Humblot, Berlin, 1835.

[Plücker 1839] J. Plücker, Theorie der algebraischen Curven: gegrundet auf eine neue Behandlungsweise der analytischen Geometrie, Adolph Marcus, Berlin, 1839.

[Poncelet 1832] J. V. Poncelet, "Théorèmes et problèmes sur les lignes du troisième ordre", Quetelet, Corr. Math. 7 (1832), 79-84. 
[Purkert 1976] W. Purkert, "Ein Manuskript Dedekinds über Galois-Theorie", Schriftenreihe für die Geschichte der Naturwissenschaft, Technik und Medezin (Berlin) 13:2 (1976), 1-16.

[Riemann 1857] B. Riemann, "Theorie der Abelschen Functionen", J. für reine angew. Math. 54 (1857), 115-155. Reprinted as pp. 88-144 in his Gesammelte mathematische Werke, Teubner, 1862; reprinted by Dover, 1953, and by Springer, 1990.

[Riemann 1862] B. Riemann, "Zur Theorie der Abelschen Functionen", pp. 487-504 in Gesammelte mathematische Werke, Teubner, Leipzig, 1862. Reprinted by Dover, 1953, and by Springer, 1990.

[Steiner 1848] J. Steiner, "Ueber allgemeine Eigenschaften der algebraischen Curven", Berichte der Akad. Wiss. zu Berlin (1848), 310-316. Reprinted in J. für reine angew. Math. 47 (1854), 1-6, and in Jakob Steiner's Gesammelte Werke, G. Reimer, Berlin, 1882 (reprinted by Chelsea, Bronx, NY, 1972).

[Valentiner 1889] H. Valentiner, De endelige transformations-gruppers theori, Danish Academy publications, series V 6, Copenhagen, 1889.

[Weber 1876] H. Weber, Theorie der Abel'schen Functionen vom Geschlecht 3, G. Reimer, Berlin, 1876.

[Weber 1896] H. Weber, Lehrbuch der Algebra, Vieweg, Braunschweig, 1896. Reprinted by Chelsea, New York, 1961.

JEREMY GRAY

FACUlty OF Mathematics

OPEN UNIVERSITY

MiLton Keynes, MK7 6AA

United KINGDOM

j.j.gray@open.ac.uk 